\title{
Chapter 19 \\ Extra Early-Flowering (exe) Mutants in Einkorn Wheat Generated by Heavy-Ion Beam Irradiation
}

\author{
Aiko Nishiura, Yusuke Kazama, Tomoko Abe, Nobuyuki Mizuno, \\ Shuhei Nasuda, and Koji Murai
}

\begin{abstract}
Four extra early-heading mutants, named extra early-floweringl (exe1), exe 2, exe 3 , and exe 4, were identified in diploid einkorn wheat (Triticum monococcum L.) following heavy-ion beam mutagenesis. Based on their phenotypes in the field, the four exe mutants were classified into two groups: Type I (moderately extra early-heading type; exel and exe3) and Type II (extremely extra early-heading type; exe 2 and exe4). Analysis of VERNALIZATION 1 (VRN1), a flowering promoter gene, showed that it was more highly expressed at earlier stages of vegetative growth in Type II mutants than in Type I mutants. Our analyses indicate that the difference in earliness between Type I and Type II mutants is associated with differences in the expression level of $V R N 1$.
\end{abstract}

\section{Introduction}

Improving our understanding of the molecular mechanisms of flowering, the phase transition from vegetative to reproductive growth associated with heading time, is one of the most important goals for wheat breeding at the present time. In bread wheat (Triticum aestivum L.), heading time is genetically determined by three characteristics: vernalization requirement; photoperiod sensitivity; and narrow-sense earliness (earliness per se). Three genes have been identified to determine the requirement of vernalization, namely VERNALIZATION 1 (VRN1), VRN2 and VRN3.

\footnotetext{
A. Nishiura • K. Murai $(\square)$

Department of Bioscience, Fukui Prefectural University, Eiheiji-cho, Fukui 910-1195, Japan

e-mail: murai@fpu.ac.jp

Y. Kazama • T. Abe

RIKEN, Nishina Center, Wako, Saitama 351-0198, Japan

N. Mizuno $\bullet$ S. Nasuda

Laboratory of Plant Genetics, Graduate School of Agriculture,

Kyoto University, Kyoto, Japan
} 
VRN1 encodes an APETALA1/FRUITFULL-like MADS-box transcription factor that is up-regulated by vernalization (Yan et al. 2003; Murai et al. 2003; Trevaskis et al. 2003; Danyluk et al. 2003). Recent studies revealed that expression of VRN1 is epigenetically suppressed in seedlings at an earlier stage of the vegetative growth phase, and that the repressive histone state is modified by the vernalization signal, leading to the up-regulation of VRN1 (Oliver et al. 2009; Diallo et al. 2012). The level of $V R N 1$ transcription gradually increases during the seedling growth stage without the need for further vernalization (Murai et al. 2003; Kitagawa et al. 2012), suggesting that the epigenetic status of VRN1 is also modified by aging. Furthermore, $V R N 1$ shows a diurnal expression pattern that is affected by daylight, with a long photoperiod producing up-regulation of its expression level (Shimada et al. 2009). In summary, these observations indicate that the VRN1 expression is also controlled by autonomous and photoperiodic pathways, as well as the vernalization pathway.

The VRN2 locus consists of two linked ZCCT genes, ZCCT1 and ZCCT2, which encode a protein with a zinc finger motif and a CCT domain (Yan et al. 2004). Natural variations have been identified in the VRN2 locus. Simultaneous deletion or non-functional mutations of these two ZCCT genes result in a plant showing the spring habit (Distelfeld et al. 2009), indicating that VRN2 is a flowering repressor gene. A high level of $V R N 2$ expression is observed in seedlings at the 1-leaf stage, while expression is down-regulated by vernalization and aging; by contrast, VRN1 shows the opposite pattern with low expression in seedlings and up-regulated expression after vernalization (Shimada et al. 2009). It has also been reported that VRN2 shows a diurnal expression pattern and that a long photoperiod up-regulates its expression level (Dubcovsky et al. 2006; Trevaskis et al. 2006), suggesting that the $V R N 2$ expression is affected by photoperiod as well as vernalization.

$V R N 3$ encodes a Raf kinase inhibitor-like protein with a high similarity to the Arabidopsis FLOWERING LOCUS T (FT) protein, which is a florigen (Yan et al. 2006). Transgenic wheat plants overexpressing VRN3 show an extra early-flowering phenotype without the need for vernalization (Yan et al. 2006; Shimada et al. 2009), indicating that $V R N 3$ is a strong flowering promoter. Under long day conditions, $V R N 3$ shows a diurnal expression pattern; however, expression is very low under short day conditions (Shimada et al. 2009; Kitagawa et al. 2012).

Based on data from expression, transgenic and mutant analyses, we developed a gene network model for the interaction of VRN1, VRN2 and VRN3 in leaves (Shimada et al. 2009). In this model, VRN1 is upstream of $V R N 3$ and activates $V R N 3$ expression under long day conditions. Thus, $V R N 1$ is proposed to play a role as an integrator of the vernalization and photoperiodic signals. Trevaskis (2010) put forward an alternative gene network model for $V R N 1, V R N 2$ and $V R N 3$; this model was based on the results of investigations using barley. This alternative model postulates that $V R N 1$ and $V R N 3$ mutually up-regulate each other: VRN1 first activates VRN3 expression, and then $V R N 3$ further activates $V R N 1$. The model was referred to as "the flowering model for temperate cereals" in a review paper on flowering in plants (Andres and Coupland 2012). More recently, a third model was suggested by Chen 
and Dubcovsky (2012). This model proposes that $V R N 1$ is activated by VRN3 and then suppresses $V R N 2$ expression. In this model, $V R N 1$ is not essential for flowering; this conclusion was drawn from an analysis of a VRN1 mutant line. However, it is not certain that the mutant line is a true VRNI knock-out, because its genotypic alteration is a point mutation and VRN1 mRNA is transcribed.

To obtain more information about the flowering mechanism in wheat, we are developing a large-scale panel of mutants induced by heavy-ion beam mutagenesis; these mutants are being systematically screened for effects on flowering time (Murai et al. 2013). Heavy-ion beam irradiation is effective at producing gene deletion mutants (null mutations) (Kazama et al. 2011, 2013). Here we describe four newly identified extra early-flowering mutant lines in diploid einkorn wheat, which have been named extra early-flowering 1 (exe1), exe2, exe 3 , and exe 4 .

\section{Identification of exe Mutants}

Seeds of the diploid einkorn wheat (Triticum monococcum $\mathrm{L}$., $2 \mathrm{n}=2 \mathrm{x}=14$, genome constitution $\mathrm{A}^{\mathrm{m}} \mathrm{A}^{\mathrm{m}}$ ) strain KU104-1 were given $50 \mathrm{~Gy}$ of $50 \mathrm{keV} \mu \mathrm{m}^{-1}$ LET (linear energy transfer) carbon ion beams and then sown in the field. The spikes of $M_{1}$ plants were bagged and the harvested selfed seeds of each spike were used to produce the $\mathbf{M}_{2}$ lines. From approximately $1,200 \mathrm{M}_{2}$ lines, we identified plants showing an abnormal extra early-heading phenotype; we termed these mutants extra earlyflowering (exe). The original wild type (WT) strain KU104-1 is a spring habit einkorn wheat having a dominant VRN1 allele and a null VRN2 allele. Therefore, the exe mutants, exe 1, exe 2 , exe 3 , exe 4 , identified in this study have no active gene at the VRN2 locus.

Table 19.1 shows the heading time of the WT and exe mutants in the field. Based on the heading time, the exe mutants were classified into two groups: Type I showed moderately extra early-heading type (exel and exe 3); and Type II showed extremely extra early-heading type (exe2 and exe4). In the field, Type I and Type II headed about 30 and 45 days earlier than the WT, respectively.

Table 19.1 Heading time of the exe mutants and wild type (WT) plants grown in the field

\begin{tabular}{l|l|l|l}
\hline Lines & Heading time & Difference from WT & Type \\
\hline WT & 6 June & - & - \\
\hline exe1 & 7 May & -30 & Type I \\
\hline exe2 & 22 April & -45 & Type II \\
\hline exe3 & 7 May & -30 & Type I \\
\hline exe4 & 25 April & -37 & Type II \\
\hline
\end{tabular}

Data from season 2011/2012 


\section{Morphological Characteristics of the exe Mutants}

WT plants and exe mutants were grown in the experimental field at Fukui Prefectural University and their morphological phenotypes were characterized during the maturation stage. Three agronomic characters were assessed: internode length, spike length, and spikelet number per spike.

The internode lengths of exe mutants were shorter than those of WT plants (Fig. 19.1). In particular, Type II exe mutants showed a significantly shorter first internode length than WT plants. Compared to WT plants, exe mutant plants produced smaller spikes with fewer spikelets (Fig. 19.2). As a consequence of the smaller numbers of spikelets, spike lengths in exe mutants were shorter than in WT plants. Furthermore, the spikes of Type II exe mutants were smaller than those of Type I mutants. These observations indicate that shortened culm lengths and fewer spikelets per spike are associated with the extra early-flowering phenotype in the exe mutants.

\section{Hypothetical Model for Extra Early-Flowering Phenotype}

The expression analysis of $V R N 1$ indicated that $V R N 1$ is highly expressed at earlier stages in Type II mutants than in Type I mutants under both short day (SD) and long day (LD) conditions (data not shown). This clearly indicates that the difference in earliness between Type I and Type II mutants is associated with the level of VRNI expression. Thus, VRN1 is essential for flowering in wheat, and the level of expression of VRN1 determines flowering time (Fig. 19.3).

Fig. 19.1 Comparison of internode lengths of exe mutants and wild type (WT) plants grown in the field. First to fourth internode lengths are shown. The mean from the main culms of five plants of each line are shown. Asterisks indicate significant $P$ values (Student's $t$-test): $* p<0.05$ and $* * p<0.001$ vs WT

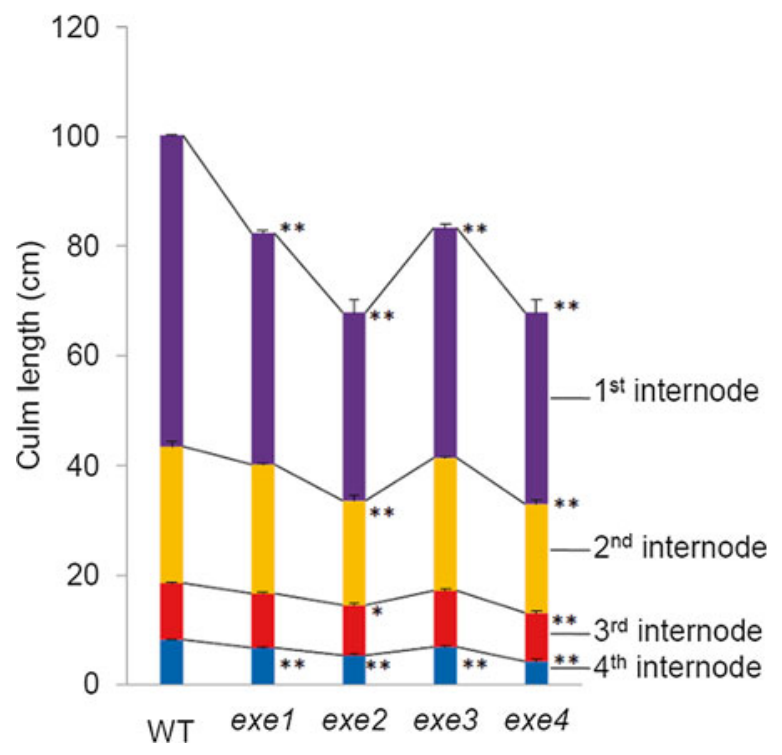


Fig. 19.2 Spikes of exe mutants and wild type (WT) plants grown in the field. The exe mutants showed a significantly decreased spike length compared to the WT

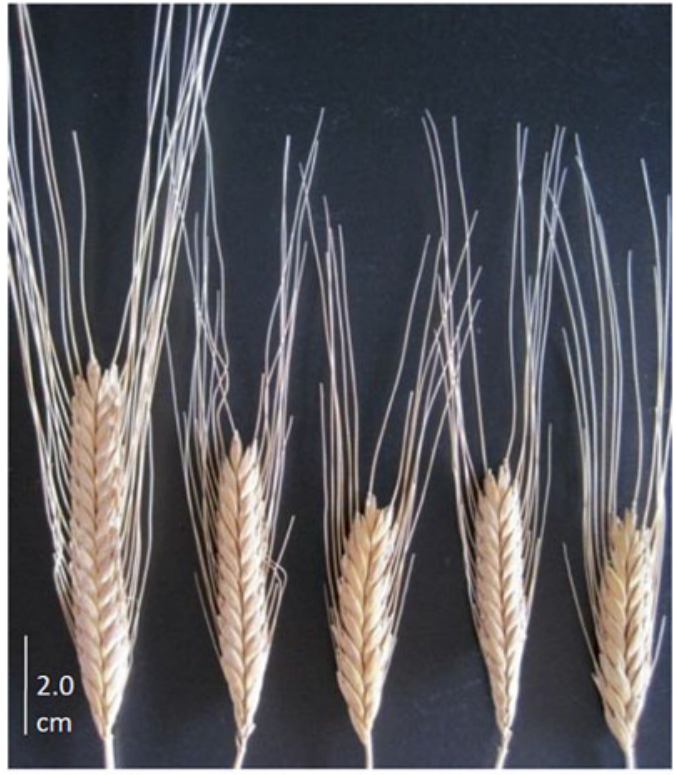

WT

exe1

exe2

exe3

exe 4

\section{Photoperiodic $\times$ Autonomous pathway pathway}

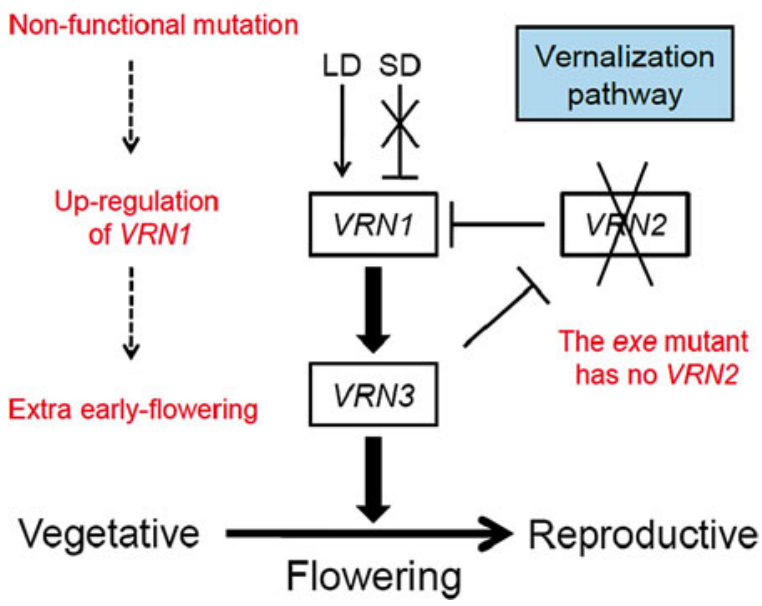

Fig. 19.3 Schematic outline of the proposed mechanism for the extra early-flowering phenotype in exe mutants. This is based on the model of Shimada et al. (2009) in which VRN1 acts as an integrator of the vernalization and photoperiodic pathways that are coordinated with the autonomous pathway. VRN1 acts by up-regulating the florigen gene VRN3. In exe mutants, the mechanism for suppressing expression of VRN1 under SD conditions must be disrupted. Levels of accumulation of $V R N 1$ transcripts induce $V R N 3$ expression, resulting in the extra early-flowering phenotype. Note that the exe mutant has no VRN2 gene, because the original strain KU104-1 lacks $V R N 2$ locus. Arrows and T-bars indicate promotion and suppression effect, respectively. Arrows indicated by bold lines show stronger effects 
Open Access This chapter is distributed under the terms of the Creative Commons Attribution Noncommercial License, which permits any noncommercial use, distribution, and reproduction in any medium, provided the original author(s) and source are credited.

\section{References}

Andres F, Coupland G (2012) The genetic basis of flowering responses to seasonal cues. Nat Rev Genet 13:627-639

Chen A, Dubcovsky J (2012) Wheat TILLING mutants show that the vernalization gene VRN1 down-regulates the flowering repressor VRN2 in leaves but is not essential for flowering. PLoS Genet 8:e1003134

Danyluk J, Kane NA, Breton G et al (2003) TaVRT-1, a putative transcription factor associated with vegetative to reproductive transition in cereals. Plant Physiol 132:1849-1860

Diallo AO, Ali-Benali MA, Badawi M et al (2012) Expression of vernalization responsive genes in wheat is associated with histone H3 trimethylation. Mol Genet Genomics 287:575-590

Distelfeld A, Tranquilli G, Li C et al (2009) Genetic and molecular characterization of the VRN2 loci in tetraploid wheat. Plant Physiol 149:245-257

Dubcovsky J, Loukoianov A, Fu D et al (2006) Effect of photoperiod on the regulation of wheat vernalization genes $V R N 1$ and $V R N 2$. Plant Mol Biol 60:469-480

Kazama Y, Hirano T, Saito H et al (2011) Characterization of highly efficient heavy-ion mutagenesis in Arabidopsis thaliana. BMC Plant Biol 11:161

Kazama Y, Fujiwara MT, Hirano T et al (2013) Characterization of a heavy-ion induced white flower mutant of allotetraploid Nicotiana tabacum. Plant Cell Rep 32:11-19

Kitagawa S, Shimada S, Murai K (2012) Effect of Ppd-1 on the expression of flowering-time genes in vegetative and reproductive growth stages of wheat. Genes Genet Syst 87:161-168

Murai K, Miyamae M, Kato H et al (2003) WAP1, a wheat APETALAl homolog, plays a central role in the phase transition from vegetative to reproductive growth. Plant Cell Physiol 44:1255-1265

Murai K, Nishiura A, Kazama Y, Abe T (2013) A large-scale mutant panel in wheat developed using heavy-ion beam mutagenesis and its application to genetic research. Nucl Inst Methods Phys Res B 314:59-62

Oliver SN, Finnegan EJ, Dennis ES et al (2009) Vernalization-induced flowering in cereals is associated with changes in histone methylation at the VERNALIZATION1 gene. Proc Natl Acad Sci U S A 106:88386-88391

Shimada S, Ogawa T, Kitagawa $S$ et al (2009) A genetic network of flowering time genes in wheat leaves, in which an APETALA1/FRUITFULL-like gene, VRN1, is upstream of FLOWERING LOCUS T. Plant J 58:668-681

Trevaskis B (2010) The central role of the VERNALIZATION1 gene in the vernalization response of cereals. Funct Plant Biol 37:479-487

Trevaskis B, Bagnall DJ, Ellis MH et al (2003) MADS box genes control vernalization-induced flowering in cereals. Proc Natl Acad Sci U S A 100:13099-13104

Trevaskis B, Hemming MN, Peacock WJ, Dennis ES (2006) HvVRN2 responds to daylength, whereas $H v V R N 1$ is regulated by vernalization and developmental status. Plant Physiol 140:1397-1405

Yan L, Loukoianov A, Tranquilli G et al (2003) Positional cloning of the wheat vernalization gene VRN1. Proc Natl Acad Sci U S A 100:6263-6268

Yan L, Loukoianov A, Blechl A, Tranquilli G et al (2004) The wheat VRN2 gene is a flowering repressor down-regulated by vernalization. Science 303:1640-1644

Yan L, Fu D, Li C, Blechl A et al (2006) The wheat and barley vernalization gene VRN3 is an orthologue of FT. Proc Natl Acad Sci U S A 103:19581-19586 